\title{
COMMUNICATION
}

\section{Complications infectieuses de l'avulsion des troisièmes molaires et carence martiale : étude rétrospective}

\author{
Guyot M', Dauzat A ${ }^{1,3}$, Enkel B',
}

1 - DCD, DM, Institut de Chirurgie Orale, Polyclinique de l'Atlantique, Saint-Herblain (France)

2 - DCD, MCU-PH, Service d'Odontologie Conservatrice et Pédiatrique, CHU de Nantes, Faculté de Chirurgie

Dentaire de Nantes (France)

3 - DCD, MAHU, Unité Fonctionnelle de Chirurgie Orale, Service d'Odontologie Restauratrice et Chirurgicale, CHU

de Nantes, Faculté de Chirurgie Dentaire de Nantes (France)

\section{Introduction}

Les cellulites post-avulsionnelles des germes de dents de sagesse ou dents de sagesse évoluées surviennent classiquement autour de la $3^{\mathrm{e}}$ semaine post opératoire d'où l'appellation " cellulites de la $3^{3}$ semaine ». Leur incidence varie de $5 \%$ à $10 \%$ des cas selon les études. Leur physiopathologie, faisant état d'une baisse des défenses physiologiques, est mal connue. L'observation d'emblée d'une prédominance féminine chez les adolescentes et jeunes adultes dans ces complications infectieuses d'une part, la fréquence des carences martiales, l'implication du fer dans les processus de cicatrisation d'autre part, nous a amené à réfléchir sur les liens potentiels entre ces différents éléments.

L'objectif de cette cohorte rétrospective est de déterminer si l'incidence des cellulites postavulsionnelles des dents de sagesse se retrouve majoritairement chez les jeunes femmes avec une carence martiale.

\section{Matériel et méthodes}

8786 dossiers médicaux numérisés de patients ayant consulté l'Institut de Chirurgie Orale durant la période du 01/01/10 au 30/06/15 ont été recensés. Nous avons inclus dans l'analyse les patients âgés de 13 à 25 ans ayant été admis pour germectomie ou avulsion de dents de sagesse. Le sexe du patient, le type d'intervention, la survenue d'une cellulite, une prescription préopératoire de ferritinémie et la présence d'une carence martiale ont été intérrogés au moyen de requêtes informatiques.

\section{Résultats}

2672 germectomies ou avulsions de dents de sagesse ont été retenues dans cette cohorte constituée de $1133(42,4 \%)$ garçons et de 1539 (57,6\%) filles. 1910 interventions ont été réalisées sous anesthésie générale (AG) et 762 sous anesthésie loco-régionale (ALR). 174 $(6,5 \%)$ cellulites post-avulsions ont été constatées entre le $20^{\circ}$ et le $30^{\mathrm{e}}$ jour post opératoire, dont $113(65 \%)$ d'entre elles chez les filles et seulement 61 (35\%) chez les garçons. Parmi les 1086 ferritinémies préopératoires prescrites, $608(56 \%)$ carences martiales ont été notées. Dans $92 \%$ des cas, les cellulites compliquent une intervention sous AG. 


\section{Discussion}

L'incidence des cellulites post-avulsionnelles dans cette cohorte rétrospective est conforme à la littérature. Elle est plus élevée chez la femme, mais il semble que la carence martiale et le type d'intervention constituent également des facteurs de risque de survenue de cette complication.

\section{Conclusion}

II serait intéressant de confirmer ces premiers résultats par une étude prospective, et confirmer l'implication de facteurs de risque comme le sexe, la carence martiale et le type d'intervention sur l'apparition de cellulites 3 semaines après germectomie ou avulsion des dents de sagesse. 